\title{
Using Contextual Pragmatics in the Teaching of Polysemy among Foreign Students
}

\author{
Melike Basbinar* \\ Tamkang University, New Taipei City 251, Taiwan Province, China \\ *Corresponding author: Melike Basbinar, melikebasbinar@gmail.com
}

\begin{abstract}
This study aims to apply contextualization in the teaching of vocabulary extensions. It argues that the introduction of vocabulary connects directly or indirectly and that systems can be found at the context level. Mandarin and "rice" were taught to two groups of international students from Asian universities; the experimental group was taught with the context system while the control group was taught with the traditional grammar translation method. The effect of contextualized teaching on vocabulary extension learning was then observed. Contextualization will benefit the learning of the extended meanings of words. An experiment was conducted to determine the learning effectiveness. The subjects of the study were international students from Asian universities, and the teaching materials were Mandarin phrases that were related to "mi" (米) and “fan” (饭) (rice). The students were divided into two groups: the experimental group was introduced the context system and the other was taught using the grammar translation method. A post-test was held to determine whether contextualized teaching improves the learning effectiveness of extended meanings.
\end{abstract}

Keywords: Contextualization; Pragmatic extension; Polysemy

Publication date: June 2021; Online publication: June 30, 2021

\section{Introduction}

An important part of language learning is the learning of meanings. In communication, once mistakes occur in the process of understanding, there would be a failure in communication. However, learning meanings is not easy, especially polysemy and idioms with special meanings, puns, and so on. Basically, the phenomenon of polysemy is common in language; a word produces polysemy, and a pun produces quotations. These quotations have often shown to be very difficult for foreign language learners, especially for semantics derived through metaphor, because they involve factors of hyperlinguistic structure, such as cultural meaning, values, and so on. Comparative semantics are non-combinatorial (non-compositional), so there are many learning methods: contextual understanding and learning, semantic disassembly, and mechanical memory. Foreign students have more difficulty in learning new words in online language.

The aim of this research is to apply theory and practice as well as the specialty of linguistics to foreign Chinese. To put it simply, the research of linguistics is mostly from vocabulary to context; however, it is believed that foreign language teaching can adopt context to vocabulary, directly teaching context architecture or system so that students can make coherent concepts and learn vocabulary meanings. It is assumed that such a way would contribute to the understanding of vocabulary extension, thus enhancing students' ability to recite vocabulary and prolonging their memory. This study attempts to apply contextualized language to foreign Chinese teaching and to observe whether contextual links are helpful for Chinese vocabulary learning (especially quotations). The main language materials are phrases related to "rice." Rice is the staple food of Chinese culture, which is the source of life, thus having a literal function. It is believed that when teaching conceptual metaphor, teachers need to have the knowledge of metaphor. 
Similarly, before teaching contextualization, it is essential to also understand the extension meanings and mutual extension relationship of "rice." In view of that, the phrases of "rice" and "meal" in Mandarin are listed to analyze and look for each extension, and then construct a context system for teaching.

\section{Contextual pragmatics and language teaching}

The relationship between context and language has been confirmed by many scholars; however, the methods of making language learning easier through contextualization are rarely mentioned. Traditional research on second language learning is mainly based on linguistics and social linguistics whereas the teaching theory and strategy of context orientation are the latest research trend. There is a relationship between context psychology and the learning of a second language. In recent years, people have begun to accept second language learning from the perspective of the context.

In 2008, Lin Kunhui combined context with language teaching and applied the context teaching method to the teaching of the concept of countable and uncountable nouns. He believes that countable and uncountable discrimination should be regarded as a conceptual activity when classifying nouns. He then derived another teaching method that is more capable in discriminating countable from uncountable nouns. His findings confirmed that context teaching does help his subjects to learn countable and uncountable nouns. In addition, they were able to remember longer than those in the grammar translation group.

Influenced by context linguistics, scholars have begun to apply conceptual metaphor to vocabulary teaching in recent years. Through experiments, it has been proven that the application of conceptual metaphor to college English vocabulary teaching can promote students' long-term memory and understanding of vocabulary, and it is an effective vocabulary teaching method. The use of conceptual metaphor has achieved great results in the teaching and learning of idioms. In 2009, Jiangfan had conducted an experiment on higher vocational students and studied the effect of conceptual metaphor teaching on the learning of foreign language metaphor vocabulary. The results showed that in short term, grammar translation teaching has similar results in helping students learn metaphor vocabulary; however, it showed significant results in terms of long-term memory. At the same time, the concept of metaphor teaching has been effective for students at varying degrees. In 2010, Ye Wenyi used English idioms to express various body parts and discussed the effectiveness of mother language translation, situational prompts, and the context teaching method. The results showed that all three teaching methods were effective but the subjects who matched the emotional classification in context of idiom memory performed well, indicating that conceptual metaphors and image base patterns assist in the understanding of foreign second language learners. However, the author believes that context teaching must also be combined with the concept of situational prompts or etymology.

\subsection{Contextualized teaching}

Institutes mainly point out the principle of language extension in context so that students can expand their semantic learning and enhance their learning of polysemy. Teaching students semantic derivative rules (metaphor) is helpful for second language learning. Once students learn that the term "bread" can be replaced by the phrase "the food one needs to survive" and the derivative "money," they would be able to draw inferences and understand that "dry bread" means crude and "bread buttered on both sides" means luck. However, teaching should not be focused only on conceptual metaphor. There are two kinds of contextualized teaching: (1) semantic quotation teaching; (2) conceptual metaphor teaching.

\subsubsection{Teaching of polysemy}

A word can have multiple different meanings; however, each semantic produced in the same context does 
not necessarily extend directly from the basic meaning. There are many ways to derivative-metaphor, metaphor or analogy, and so on. The theory of grammatology and grammatization can be used to determine the relationship between multiple semantics and possible quotation processes. By putting it into the context, it can clearly present the source of the extension and the cause of it, thus helping foreign students to learn.

\subsubsection{Conceptual metaphor}

Conceptual metaphor teaching mainly introduces the theory of conceptual metaphor so that the students would be able to appreciate how metaphors operate in context and are reflected in language. Basically, conceptual metaphor is not just an image metaphor, but the mapping of the whole system. When "armor" is compared to "ethylene" in concept, the relevant terms of ethylene may appear in the field of armor and be used with it. When students learn the conceptual metaphor of "a rice bowl is a job," they would be able to appreciate that some work-related words are related to "rice bowl."

\section{Example:}

Find a job = Find a "rice bowl"

No job = No "rice bowl"

Getting a job = Getting a "rice bowl"

Losing a job = Losing a "rice bowl"

In this way, students can improve their ability to make sentences. At the same time, students will also be able to associate with the material of the rice bowl and the nature of the work.

\section{Extension of semantic meaning}

In order to communicate efficiently, it is essential to know that there are many polysonyms in a language. Synonyms refer to words that have several interrelated meanings. According to the encyclopedia on Baidu, there are basic meanings (original or common meanings), extension meanings (from basic meanings), and quotation meanings (meanings that are fixed by using basic meanings to other things).

How do meanings extend? From the above definitions, it can be appreciated that the way language meanings extend involves quotations and metaphors. The encyclopedia on Baidu also mentioned that polysemy words are often related to metaphors, borrowing, puns and other rhetorical techniques. In 2014, Liu and Lian studied the polysemy of "walking" in Hokkien and mentioned that polysemy can produce more extension through inferences, metaphors, and implicit sessions. Generally, the common ways that meanings extend include quotation, metaphor, borrowing, analogy, and so on. However, quotations and metaphors are difficult to distinguish. There are many ways of changing word meanings, among which the most important is "extension," where the type of extension has a figurative extension. Therefore, quotation meanings are also extended meanings. According to Li Ashu, introduction is a fixed meaning formed through figurative usage. In other words, metaphor is also the meaning of the deduction and extension of the meaning, which is an aspect of the extension. "Borrowing" is to substitute another, basically a metaphor. Analogy (or simulation) transfers the context of a specific thing to other specific things, where both do not necessarily have substantial homology, and their simulation is unreasonable. "The analogy of hacks is sometimes a simulation, such as comparing the moon to silver coins," as mentioned in Baidu. Therefore, there is overlap between analogy and metaphor. Since most of the approaches are related to metaphors, there are many scholars who study polysonyms, focusing on quotations or classifying all quotations as figurative. Although some quotations do not necessarily come directly through metaphor, there may be indirect relations in the context structure, such as the analogy from metaphor. Therefore, the focus is not 
only on the part of the conceptual metaphor, but to provide the whole meaning-derived correlation diagram to clearly present the context system.

A large number of polysemies satisfy the economic principles of the language to reflect infinite objective things with limited words, but also create certain difficulties in the understanding and use of the language. The phenomenon of "one word but with multiple meaning" is a major obstacle for foreigners in learning Mandarin. The interpretation in the general dictionary of polysemy has the same concept of putting all meanings under one word so that it only provides the context to the learners, not to derive many meanings from a word. This study seeks to identify the thinking paths of many semantic terms and teach vocabulary using extended context network maps.

\subsection{Meanings and language extension of "rice"}

Language refers to a series of extended vocabulary involved in the staple food of the culture. There is a figurative phenomenon of food-related words in Hokkien proverbs. According to a research, food-related words in Hokkien can symbolize the status of the rich and the poor, mainly with their staple: rice and grain. Related to the cultural background, Taiwan is an agricultural country, where rice is the staple of the people there; thus, rice has a rich metaphor.

\section{Example:}

Big monkey throwing rice (Wastage)

No rice and leap month (Money is needed to make ends meet)

Three generations of grains, emptied at once (Rich but only for three generations)

Food, firewood, and rice (A metaphor for economic difficulties)

Trouble with thirteen generations of cooking (The satirist thinks too much)

In 2009, Ding studied the relationship between language expansion and culture, mentioning that the attitude of English toward staple food is similar to that of Chinese toward rice. Bread has "livelihood and income."

\section{Example:}

To earn one's bread (To make a living)

Bread and butter (Basic means of income)

Bread and cheese (The barest necessities of life)

To take bread out of one's mouth (To remove one's livelihood)

To know which side one's bread is buttered (To be mindful of one's own interest)

Bread and water (A simple meal)

In Chinese, rice is not just rice. There are other quotations besides referring rice as the aforementioned. In Hometown, it was mentioned, "In ancient times, and even modern times, rice is Fulu, rice is gold, rice is education, rice is wealth." Wealth was very commonly referred to in ancient Chinese society. At that time, the salaries of the government officials were measured by a few stone meters. Tao Yuanming's "five buckets of rice" refers to a meager salary. According to the State's Education Dictionary, the following phrases and idioms about "rice" and "meal" have been found:

"Rice rotting in a warehouse" describes riches.

"Waiting for rice" means economic difficulties or waiting for money.

"Rice bowl" means relying on a living. 
"Mixed meal" is described as a meager amount and barely sustainable.

"Eat" means to keep on living; for example, "He 'eats' by teaching."

"Eating guy" means living tools or skills.

"Enjoy food" means to ask others for food. Today, it is a metaphor of working.

"A bite of rice" is similar to a meager treatment or food.

Basically, "rice" refers to money, work, family planning, economic conditions, and so on. Among them, money has the most direct relationship with "rice" because money can be used to buy rice. Therefore, "rice" in the concept of language expansion is equivalent to money. Working to have money (salary) as well as earning money to support the family and home planning are indirectly associated with "rice" through the direct relationship with money. The container can often represent its content, such as the bottle representing the wine inside and the rice bowl referring to the rice in it. The direct relationship of "rice bowl" with rice is the basis for it having an indirect meaning of "work," "money," "family planning," and so on.

\section{Research and discussion}

After constructing the extension of "rice," an experiment was carried out to observe whether the effect of contextualization is the same as assumed.

\subsection{Experimental design}

\subsubsection{Subjects}

The study included ten foreign students from Asian universities, five being Mongolian and another five being Indonesian. These students were able to speak Mandarin a little, some of which were better, and scored quite high in their tests. The Indonesian students were less familiar with Mandarin compared to their Mongolian counterparts.

\subsubsection{Methods}

The students were requested to translate Mandarin sentences, which could be used in Mandarin or English. There were 10 sentences, five of which were phrases related to "rice," while the other five were idioms that were once taught in their classes without any relation to the term, "rice." Another 10 sentences were used in the post-test, seven of which were phrases related to "rice," and the other three were idioms that were once taught in their classes without any relation to the term, "rice." Two out of the seven rice-related phrases were the same as the pre-test while the other five were new phrases. The two identical phrases were to observe the students' memory skills, while the five new phrases were to determine the students' ability to understand. The 10 sentences in the post-test were used to test the students again two months later.

\subsubsection{Assumptions}

It was assumed that the memory and language skills of the contextualized language group would be better than those of the grammar translation group. The main reason behind such an assumption is because humans generally have some common experiences with similar conceptual structures at the contextual level that can produce similar linguistic usage. Eating is a common human experience, and staple food is an essential to survive. Therefore, human beings have the same view on staple food. That is to say that different languages choose different words, but the context system is the same as the conceptual metaphor. In this way, the staple food of each culture will have similarities. In addition to common experience, different experiences can also be applied in contextualized teaching. This allows students to understand the thinking 
patterns of different cultures while learning foreign languages.

\subsubsection{Procedure}

The test was conducted before teaching. Two days after the test, the students in both groups were taught. The experimental group was taught contextualization to understand how "rice" is related to work, income, and money. For the students in the control group, the traditional way of translating and explaining the five rice-related phrases that were seen in the previous test were used. The post-test was conducted on the day after the teaching. More than a month after the winter vacation, the study continued to observe which group of students had longer memory.

\subsection{Results and discussion}

As the test only required semantic translations, the students were not required to translate the whole sentence. In view of that, the answer rate was calculated only if the phrase pertaining "rice" was correct and not the whole sentence. The first two shown in the following table (Table 1) were phrases that appeared in both the pre-test and post-test, which were taught. The last five were phrases that appeared in the posttest, which were not taught. "Snatching the rice bowl" (抢饭碗) appeared in the pre-test but not in the posttest. It can be seen from the answer rate that students understood the term, "rice bowl," even before the teaching session.

Table 1. Pre-test and post-test answer rates of the experimental group and control group

\begin{tabular}{ccccc}
\hline & \multicolumn{2}{c}{$\begin{array}{c}\text { Experimental group } \\
\text { (Contextualized teaching method) }\end{array}$} & $\begin{array}{c}\text { Control group } \\
\text { (Grammar translation teaching method) }\end{array}$ \\
\cline { 2 - 5 } & Pre-test correct rate & Post-test correct rate & Pre-test correct rate & Post-test correct rate \\
\hline 没饭吃 & $100 \%$ & $100 \%$ & $100 \%$ & $75 \%$ \\
赏一口饭 & $33 \%$ & $100 \%$ & $0 \%$ & $25 \%$ \\
抢饭碗 & $66 \%$ & & $50 \%$ & \\
吃这行饭 & & $100 \%$ & & $50 \%$ \\
吃饭家伙 & & $66 \%$ & & $100 \%$ \\
长期饭票 & & $66 \%$ & $50 \%$ \\
铁饭碗 & & $100 \%$ & $25 \%$ \\
丟饭碗 & & $100 \%$ & & $25 \%$ \\
\hline
\end{tabular}

Through preliminary observation, students who received contextualized teaching achieved better results for the taught rice-related phrases and the correct rates were also relatively higher. In regard to the new phrases, it can be seen that the students under the contextualized teaching method also performed well. Although the control group had learned the meaning of "snatching the rice bowl" (抢饭碗) during their lessons, their correct rates to “iron rice bowl” (铁饭碗) and “lose a rice bowl” (丢饭碗) were low, indicating that the grammar translation teaching method did not improve their ability to draw inferences from one example. From the study, there are several interesting phenomena. For “long-term meal ticket” (长期饭票), only one student from the control group answered correctly, while all the other students wrote "long-term stable work." This shows that the students had no problem with associating "rice" with work, the problem was the difference in culture. Therefore, it can be concluded that culture has some influence on lexical extension. In addition, for the two rice phrases taught in the lesson, the correct rates of the control group in the post-test were lower than the pre-test. It can be argued that such a phenomenon implies situations where 
traditional methods are prone to result in memory instability.

\section{Conclusion}

This study attempted to apply the results of linguistics to the teaching of Chinese among foreign students. There have been many methods of teaching Chinese to foreign students but generally, the grammar translation teaching method has been used for vocabulary teaching. In recent years, people have started to adopt the conceptual metaphor teaching. This research proposed the contextualized teaching method, arguing that conceptual metaphor teaching must be matched with the context teaching of semantic extension. A preliminary experiment was carried out on ten foreign students from Asian universities, and the results complied with the hypothesis, confirming that contextualized teaching can improve the learning effect in introducing Chinese vocabulary. However, with the small number of students, further research should be carried out to gather more accurate results.

The effect of contextualized teaching deserves recognition and attention; however, the traditional grammar translation teaching method must still exist because not all words have quotations, and some idioms still need to be learned through the traditional way. Basically, contextualized language teaching is suitable for the vocabulary of systematic extension network and conceptual metaphors in the concept of contextual context.

\section{Disclosure statement}

The author declares that there is no conflict of interest.

\section{References}

[1] Brugman C, 1981, The Story of 'Over': Polysemy, Semantics and the Structure of the Lexicon (MA Thesis). University of California, Berkeley (published New York: Garland, 1988).

[2] Chen Y, 2018, Prosodic Comparisons of Two Types of Realization of Focus in Mandarin. Proceedings of the 9th International Conference on Speech Prosody 2018, 917-921.

[3] Carrió-Pastor ML, 2009, Content and Language Integrated Learning: Cultural Diversity, Linguistic Insights: Studies in Language and Communication.

[4] Evans V, 2005, The Meaning of Time: Polysemy, the Lexicon and Conceptual Structure. J Linguistics, 41: 33-75.

[5] Glynn D, Robinson JA, (eds) 2014, Corpus Methods for Semantics: Quantitative Studies in Polysemy and Synonymy, John Benjamins, Amsterdam.

[6] Rothstein SD, 2008, Theoretical and Crosslinguistic Approaches to the Semantics of Aspect, Linguistik Aktuell. 